\title{
Influence of disorder and a parallel magnetic field on a Quantum Cascade Laser
}

\author{
V.M. Apalkov and Tapash Chakraborty \\ Max-Planck-Institut für Physik Komplexer Systeme, 01187 Dresden, Germany
}

\begin{abstract}
The luminescence spectra of a quantum cascade laser in a strong magnetic field is influenced significantly by the presence of disorder (charged or neutral) in the system. An externally applied magnetic field parallel to the electron plane causes a red shift of the luminescence peak in the absence of any disorder potential. Our results indicate that the disorder potential tends to cancel that red shift and causes a rapid decrease of the luminescence peak. A similar behavior was observed in a recent experiment on QCL in a parallel magnetic field.
\end{abstract}

Since the invention of the unipolar semiconductor quantum cascade laser (QCL) in 1994 [1,2], there have been a series of innovations and improvements of performances in this field of intersubband light sources [3, 8 . The QCL has already established its potential as a tunable, high-power coherent source in the mid-IR spectral range, and therefore amenable to a host of very useful applications [8]. In the quest for further improvements of the QCL device, magnetic field studies have recently been performed $[9] 10$. However, no experimental or theoretical studies are available yet on the role of disorder in the optical properties of the QCL. Although the techniques of crystal growth have improved significantly in recent decades, interface roughness in QCL structures cannot be entirely eliminated. In a narrow quantum well, electrons are closer to the interface and are influenced by the irregularities or by the charged impurities confined at the interface. In this letter, we report on our findings that the effect of disorder is particularly significant on the luminescence spectra in the presence of a magnetic field. The QCL structure we have studied here is sketched in Fig. 1 [11], where the parameters are taken from the QCL structure reported in [9].

The main results of the experimental work on the optical spectra of a QCL in a strong parallel magnetic field [9] were, a small blue shift of the emission peak, decrease of the emission intensity and broadening of the peak in a strong magnetic field. It was also found that the singleparticle theoretical model can not explain these observations [9] [10]. This model predicts a red shift of the peak that can not be cancelled by the many-body corrections [11]. In order to explain the experimentally observed tiny blue shift of the emission line, we consider the singleparticle Hamiltonian

$$
\begin{aligned}
H= & \frac{p_{z}^{2}}{2 m(E)}+\frac{1}{2 m(E)}\left(p_{x}+\frac{e}{c} B z\right)^{2}+\frac{p_{y}^{2}}{2 m(E)} \\
& +V_{\text {conf }}(z)+V_{\text {dis }}(x, y)
\end{aligned}
$$

where the magnetic field $B$ is applied in the $y$-direction, the vector potential is $\vec{A}=(B z, 0,0), V_{\text {conf }}(z)$ is confinement potential due to conduction-band discontinuity and bias electric field (Fig. 1), and $V_{\text {dis }}(x, y)$ is the disorder potential. The effective mass of the particle depends on the energy due to the band nonparabolicity. We consider

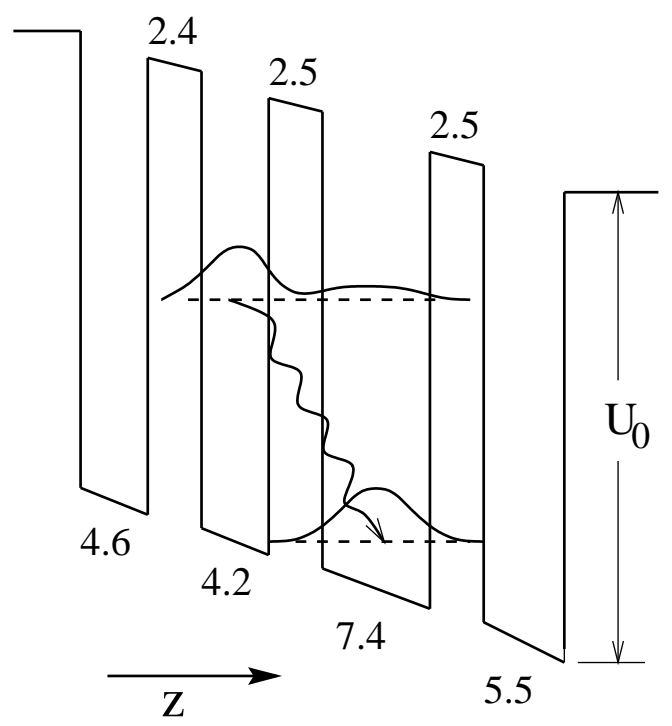

FIG. 1. Energy band diagram (schematic) of the active region of a quantum cascade laser structure under an average applied electric field of $55 \mathrm{kV} / \mathrm{cm}$. Only one period of the device is shown here. The relevant wave functions (moduli squared) as well as the transition corresponding to the laser action are also shown schematically. The numbers (in $\mathrm{nm})$ are the well $\left(\mathrm{Ga}_{0.47} \mathrm{In}_{0.53} \mathrm{As}\right)$ and barrier $\left(\mathrm{Al}_{0.48} \operatorname{In}_{0.52} \mathrm{As}\right)$ widths. Material parameters considered in this work are: electron effective mass $m_{e}^{*}\left(\mathrm{Ga}_{0.47} \operatorname{In}_{0.53} \mathrm{As}\right)=0.043 m_{0}, m_{e}^{*}$ $\left(\mathrm{Al}_{0.48} \operatorname{In}_{0.52} \mathrm{As}\right)=0.078 m_{0}$, the conduction band discontinuity, $U_{0}=520 \mathrm{meV}$, the nonparabolicity coefficient, $\gamma_{w}=1.3 \times 10^{-18} \mathrm{~m}^{2}$ for the well and $\gamma_{b}=0.39 \times 10^{-18}$ $\mathrm{m}^{2}$ for the barrier, and the sheet carrier density induced by doping, $n_{s}=2.3 \times 10^{11} \mathrm{~cm}^{-2}$. The energy difference between the two levels where the optical transition takes place, is 132 meV. 
the same disorder potential for both quantum wells in the active region (Fig. 1), i.e., electrons feel the same potential before and after emission.

We have studied the single-particle energy spectra and emission lines for two types of disorder potentials: for disorder due to the surface roughness in quantum wells and for disorder due to the doped charged impurities. The disorder potential in the first case is described by the potential $V_{\mathrm{dis}, 1}(x, y)$ which has zero average and Gaussian correlations

$$
\left\langle V_{\operatorname{dis}, 1}\left(\vec{r}_{1}\right) V_{\text {dis }, 1}\left(\vec{r}_{2}\right)\right\rangle=V_{0}^{2} \mathrm{e}^{-\left(\vec{r}_{1}-\vec{r}_{2}\right)^{2} / l_{0}^{2}}
$$

where $\vec{r}$ is the two-dimensional vector.

In the case of a charged impurity we assume that all impurities are in the same two-dimensional plane with position $z_{\text {imp }}$. The potential has the form

$$
V_{\mathrm{dis}, 2}(\vec{r})=-\sum_{i} \frac{e^{2}}{\epsilon} \frac{\mathrm{e}^{-\left|\vec{r}-\vec{R}_{i}\right| / d_{\mathrm{sc}}}}{\sqrt{\left(\vec{r}-\vec{R}_{i}\right)^{2}+\left(z-z_{\mathrm{imp}}\right)^{2}}}
$$

where $\vec{R}_{i}$ is the two-dimensional vector describing the position of $i$-th impurity, $d_{\mathrm{sc}}$ is the phenomenological constant which describe the screening of impurity potential by electrons. In our present calculations we used $d_{\mathrm{sc}}=10$ $\mathrm{nm}$. The two-dimensional density of impurities is equal to $n_{\mathrm{imp}}=2.3 \times 10^{11} \mathrm{~cm}^{-2}$. The positions of impurities are uncorrelated.

To find the emission spectra of the system with the disorder potential $V_{\text {dis }}(x, y)$ we calculate from Hamiltonian (11) but without the disorder potential, the dispersion of the electron on the first and on the second subband (Fig. 1 ) for a given magnetic field $B$. Without any disorder the states are characterized by the two-dimensional momentum, $\left(\hbar k_{x}, \hbar k_{y}\right)$. Taking these states as a basis we diagonalize the total Hamiltonian with the given disorder potential in a square geometry of size $L$ with periodic boundary conditions. The emission spectra are then found from

$$
\begin{aligned}
I(\omega)= & \left\langle\sum_{i f} \delta\left(\omega-E_{i}+E_{f}\right)\left|\int \xi_{2}(z) \xi_{1}(z) d z\right|^{2}\right. \\
& \left.\times\left|\int \psi_{i}^{*}\left(k_{x}, k_{y}\right) \psi_{f}\left(k_{x}, k_{y}\right) d k_{x} d k_{y}\right|^{2}\right\rangle
\end{aligned}
$$

where $E_{i}, E_{f}$ are electron energies before and after emission; $\xi_{1}(z), \xi_{2}(z)$ are wave functions of the electron on the first and second subbands; $\psi_{i}\left(k_{x}, k_{y}\right), \psi_{f}\left(k_{x}, k_{y}\right)$ are the initial and final wave functions for a given disorder potential. The summation in (2) is over all final states and over occupied initial states. The number of occupied initial states is determined by $N_{i}=L^{2} / n_{\mathrm{el}}$, where $n_{\mathrm{el}}=2.3 \times 10^{11} \mathrm{~cm}^{-2}$ is the electron density. The anglular brackets correspond to averaging over disorder potential.

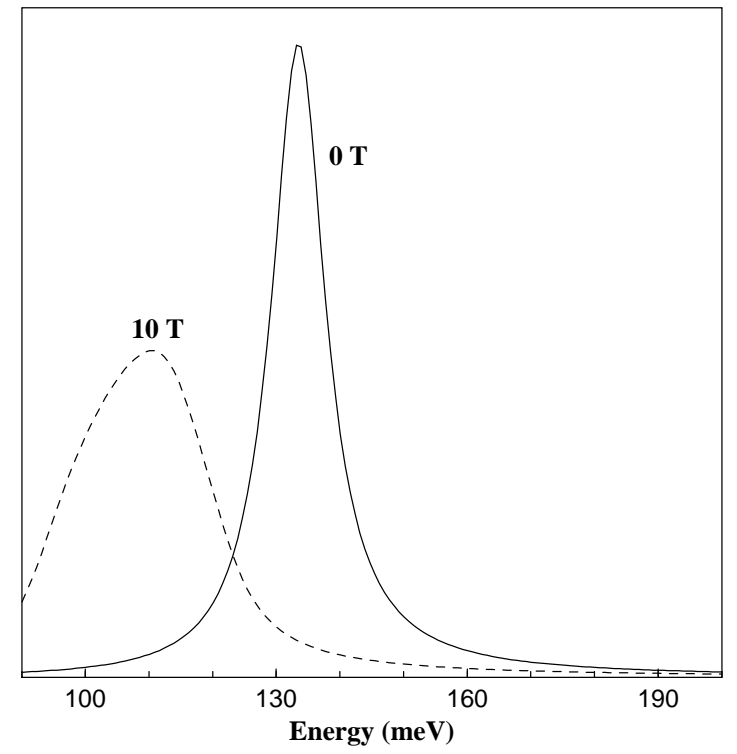

FIG. 2. Luminescence spectra of a quantum cascade structure in the presence of an externally applied parallel magnetic field, but in the absence of any impurity.

To understand the properties of the emission line of our system with disorder we consider the first terms of a perturbation theory in a magnetic field [12]. In this case the Hamiltonian (1) for subband $\alpha$ can be rewritten as

$$
H_{\alpha}=\frac{1}{2 m(E)}\left(p_{x}+\frac{e}{c} B\langle z\rangle_{\alpha}\right)^{2}+\frac{p_{y}^{2}}{2 m(E)}+V_{\mathrm{dis}}(x, y)
$$

where $\langle z\rangle_{\alpha}=\int \xi_{\alpha}(z) z \xi_{\alpha}(z) d z$ and we disregard the small diamagnetic term. In this case the energy spectra in the non-zero magnetic field is the same as for the zero magnetic field and the wave functions differ only by a phase factor which correspond to a translation in $k$ space of the vector $\left(\frac{e B}{\hbar c}\langle z\rangle_{\alpha}, 0\right)$. The main effect is on the emission spectra because the intensity of optical transition from the initial state $i$ to the final state $f$ is proportional to the overlap between $\psi_{i}\left(k_{x}, k_{y}\right)$ and $\psi_{f}\left(k_{x}-k_{B}, k_{y}\right)$, where $\psi_{i}$ and $\psi_{f}$ are the wave functions of the initial and final system respectively, in the absence of a magnetic field

$$
\mathcal{I}_{i f}=\left|\int \psi_{i}^{*}\left(k_{x}, k_{y}\right) \psi_{f}\left(k_{x}-k_{B}, k_{y}\right) d k_{x} d k_{y}\right|^{2} .
$$

The wave vector $k_{B}$ is equal to

$$
k_{B}=\frac{e}{\hbar c} B\left(\langle z\rangle_{i}-\langle z\rangle_{f}\right)
$$

and is of the order of $k_{B} \sim 0.1 \mathrm{~nm}^{-1}$ at $B=10$ tesla for the system parameters considered here (Fig. 1). 

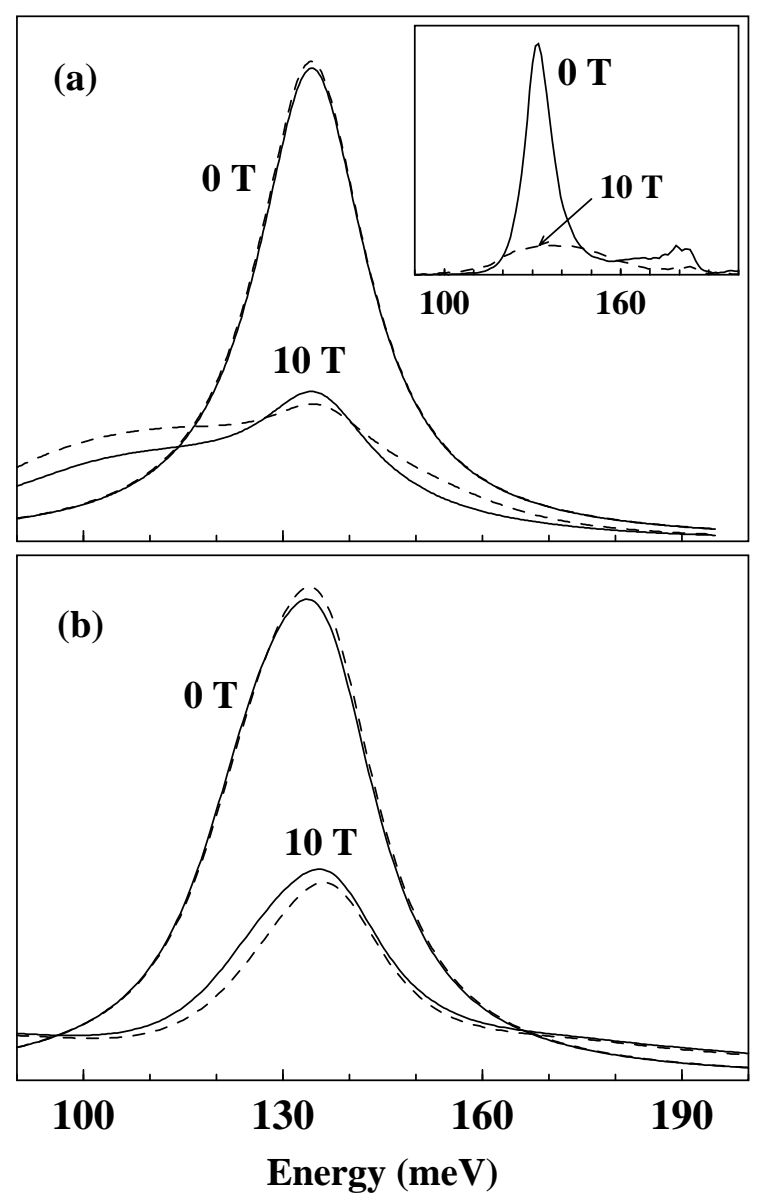

FIG. 3. Luminescence spectra of a quantum cascade structure in the case of (a) charge-neutral impurities and (b) charged impurities in the presence of an externally applied parallel magnetic field. The experimental results of Ref. [9] are shown as inset.

The position of the maximum of the emission line is determined mainly by the transitions from the ground state of the single-particle system. Without any disorder, the single-particle wave functions are plane waves $\psi_{i}\left(k_{x}, k_{y}\right)=\delta\left(k_{x}-k_{x, i}\right) \delta\left(k_{y}-k_{y, i}\right)$, with the ground state at $\vec{k}=0$. Clearly, the allowed transitions are then only from the ground state $\left(k_{x}=0\right)$ of the upper subband to the excited states $\left(k_{x}=k_{B}\right)$ of the lower subband, resulting in a red shift of the emission line in a magnetic field. A strong enough disorder will localize the lowest states. If the localization length is smaller than $1 / k_{B}$ then the overlap between the initial ground state to the final ground state is large and transition from a ground state to a ground state (different subbands) of the system is strong in a magnetic field and the red shift of the emission line is suppressed.

In Fig. 2, the emission spectra are shown for the system without disorder for two values of the magnetic fields. The large red shift, as expected, is clearly seen in the figure. In Fig. 3, the emission spectra are shown for the system with disorder. In Fig. 3a, where the disorder is charge neutral, the solid curves correspond to $V_{0}=30$ $\mathrm{meV}$ and $l_{0}=5 \mathrm{~nm}$, and the dashed curves correspond to $V_{0}=20 \mathrm{meV}$ and $l_{0}=5 \mathrm{~nm}$. In Fig. $3 \mathrm{~b}$, the disorder is due to the charged impurity and the solid and dashed lines correspond to separation between the impurity plane and $4.2 \mathrm{~nm}$ quantum well (Fig. 1) to be 3 and $4 \mathrm{~nm}$, respectively. In both cases we see large suppression of the red shift observed in Fig. 2. The emission line for the high magnetic field case is almost at the same energy as for the zero-field case, and in Fig. 3b there is a small blue shift of the peak. These results are qualitatively in agreement with experimental data of [9, 10] and shown as inset in Fig. 3. Further, in Fig. 3 the intensity of the luminescence peak shows a rapid decrease in the presence of disorder and high fields because of small overlap in Eq. (3).

The small blue shift in Fig. $3 \mathrm{~b}$ is due to the band nonparabolicity. At zero magnetic field the nonparabolicity of band structure allows the transitions from the ground state to the exited states. The magnetic field redistributes the transitions and increase the intensity of transition into the ground state which results in small blue shift in Fig. 3b. From our results it is clear that in a QCL device, magnetic field induced luminescence spectra 11 can only be observed in the absence of strong disorder, i.e., devices with high electron mobility or for wider quantum wells.

We thank P. Fulde for his support and kind hospitality in Dresden and Stéphane Blaser for valuable discussions on his experimental results.

[1] J. Faist et al., Science 264, 553 (1994).

[2] J. Faist et al., Appl. Phys. Lett. 66, 538 (1995).

[3] J. Faist, et al., Appl. Phys. Lett., 72, 680 (1998).

[4] J. Faist, et al., Appl. Phys. Lett., 683680 (1996).

[5] J. Faist, et al., IEEE Photon. Technol. Lett., 10, 1100 (1998).

[6] C. Sirtori et al., Appl. Phys. Lett. 73, 3486 (1998).

[7] G. Strasser et al., Appl. Phys. Lett. 75, 1345 (1999).

[8] Proceedings of the Fifth International Conference on Intersubband Transitions in Quantum Wells, editors, M. Helm, K. Unterrainer, Physica E 7 (2000).

[9] S. Blaser, et al., Physica E 7, 33 (2000).

[10] S. Blaser, et al., unpublished results.

[11] V. Apalkov and T. Chakraborty, to be published, condmat/0008238.

[12] W. Beinvogl, A. Kamgar, and J.F. Koch, Phys. Rev. B 14, 4274 (1976). 